Beispiel: Der Caleiumcarbonatgehalt (18\%) in obigem Backpulver ist zu bestimmen. $1,5 \mathrm{~g}$ Substanz verbrauchen $2 \pi \mathrm{r}$ Neutralisation $10,5 \mathrm{ccm} \quad 1 / 4 \mathrm{~N}$.-Natronlauge. Nach der Auffüllung auf $200 \mathrm{ccm}$ werden zu $50,0 \mathrm{ccm}=0,375 \mathrm{~g}$ Substanz $10,0 \mathrm{ccm} 1 / 4$ N.-Salzsäure zugesetzt und gekocht; zum Zurücktitrieren werden $6,2 \mathrm{ccm} \quad 1 / 4 \mathrm{~N}$.-Natronlauge verbraucht. Die Differenz $=\frac{3,8 \times 0,021 \times 100}{0,375}$ ergibt $21,3 \%$ Natriumbicarbonat mit $11,16 \%$ Kohlendioxyd.

Gesamt-Kohlendioxyd in $17 \mathrm{~g}$ (nach oben $3,22 \mathrm{~g}$ ) . . = 18,95\% Kohlendioxyd

$\mathrm{Ab}$ Kohlendioxyd von gefundenem Natriumbicarbonat $11,16 \%$

$7,79 \%$ Kohlendioxyd

$=17,7 \%$ Calciumcarbonat.

Als Indicator ist Phenolphthalein zu verwenden; Methylorange würde wegen seines völlig verschiedenen Verhaltens gegenüber den Phosphaten gänzlich unrichtige Werte ergeben.

Die Fehler dieser Bestimmungsweise liegen auf der Hand; beim Finschütten des Pulvers in das Wasser wird schon eine leichte Kohlensäureentwickelung stattfinden, rasches Neutralisieren ist deshalb nötig; ferner ist der Endpunkt dieser Neutralisation nicht sehr scharf, zumal die sauren Bestandteile erst während der Neutralisation in Lösung gehen, was aber andererseits auch eine Verzögerung in der Einwirkung auf das Bicarbonat zur Folge hat. Indem man durch den Vorversuch die annähernde Menge Alkalilange feststellt, wird der Fehler zum großen Teil ausgeschaltet. Hat man etwas reichlich Alkalilauge zur Neutralisation verwendet, so wird dies durch die nachfolgende starke Verdünnung wieder ausgeglichen.

Die erbaltenen Resultate für den Natriumbicarbonatgehalt können bei einiger Übung um 0,5 bis höchstens $2 \%$ von der Richtigkeit abweichen; dies erscheint beträchtlich, ist aber in Anbetracht der Schwierigkeit bezw. Unmöglichkeit einer genauen Bestinmung des ursprünglichen Bicarbonat- und Calciumcarbonatgehaltes eines Backpulvers noch als zufriedenstellend anzusehen.

\title{
Über die Verwendung von Robiniensamen als Nahrungsmittel.
}

\author{
Von
}

Willibald Hanikirsch in Leitmeritz.

[Eingegangen am 1. Juli 1918.]

Professor Nestler machte mich unter Bezugnahme auf eine Notiz in der Zeitschrift für angewandte (hemie ${ }^{1}$ ) auf die Möglicbkeit aufmerksam, daß man bei der Untersuchung von Kaffeesurrogaten usw, auf Samen von Robinia pseudacacia stoßen könnte. Da gerade über den anatomischen Bau derselben und ähnlicher stark giftiger Samen z. B. Cy tisus Laburnum (Goldregen) noch wenig spezielles bekannt ist und auch sonst dem Nahrungsmittelchemiker keine Handhabe zur Erkennung derselben gegeben ist, so entschloB ich mich, mich mit dieser Sache etwas näher zu beschäftigen.

Die Notiz in der oben genannten Zeitschrift lautete: "Der Kriegsausschub hat zum Sammeln von Akaziensamen erst jetzt aufgerufen, nachdem es der Firna Zuntz sel. Wwe. gelungen ist, aus den mit Benzin extrahierten Rückständen einen Kaffee-Ersatz bezw. Kakao-

1) Zeitschr. angew. Chem. 1917, 30, 206. 
Ersatz herzustellen, sodaßs die Akaziensamen, abgesehen von dem aus ihnen gewinnbaren, genufiäähigen Öle, in doppelter Weise der menschlichen Ernährung nunmehr nutzbar gemacht werden können."

Auch brachte ich in Erfahrung, daf andere Firmen bereits an eine Verwendung von Robiniensamen zu Kaffeesurrogaten gedacht haben, und als ich bereits meine Arbeit abgeschlossen hatte, bekam ich C. Griebel's $\left.{ }^{1}\right)$ Arbeit über Kaffee-Ersatzstoffe zur Hand, die jedoch insoferu auf diese Arbeit obne Einflua blieb, als GriebeI den Samen von Robinia nicht eingehend genug beschrieb und auch nicht auf die Beschreibung ähnlicher Samen einging.

Bei näherer Umschau sehen wir, daß der genannte Baum sehr häufig bei uns angepflanzt ist.

Die Gattung Robinia aus der Familie der Papilionaceen ist in Nordamerika von Pennsylvanien bis Nordgeorgien heimisch und bildet 6 gut bekannte und mehrere zweifelhafte Arten. Die bekanntesten sind Robinia pse udacacia L., die gemeine Akazie, mit Trauben ohne Borsten, Blütenkrone weiß oder hellrosa überlaufen; ferner Robinia glutinosa Sims, mit schwach rosafarbenen Blïten, Robinia neomexicana A. Gray, deren Blüten nicht in Trauben herabhängen, sondern in Dolden aufrecht stehen und die namentlich in Kolorado, Utah, Arizona und Neumexiko auftritt, und endlich Robinia hispida L., die borstige Akazie, die wehrlos ist und aus dem südōstlichen Amerika stammt.

Diese 4 bekanntesten Arten findet man überall bei uns als Zierbäume angepflanzt, am häufigsten Robinia pseudacacia in mehreren Formen, auch verwildert. Ioh will noch kurz diese Formen erwähnen: Als Straßen- oder Alleebaum viel verwendet ist Robinia pseudacacia Bessoniana oder inermis, die Kugelakazie; beide Formen bilden ihre Kronen zu einer Kugel aus, ohne daß ein Schneiden notwendig wäre. Der Baum blüht aber sehr selten, meist überhaupt nicht und kommt daher zum Sammeln der Samen nicht in Betracht. Ein Baum von eigenartiger Sehönheit ist Robinia pseudacacia pendula, die Trauerakazie, mit dicht belaubten, bis auf den Boden herabhängenden Zweigen. Die als Zierbaum wertvollste Form ist aber Robinia pseudacacia Decaissneana mit großem Blütenreichtum und prächtiger Blütenschönheit, Formen, die man bei uns seltener antrifft, sind noch Robinia pseudacacia angustifolia elegans mit einer zarten Belaubung, die gelbblätrige Akazie, Robinia pseudacacia aurea, ferner Robinia pseudacacia pyramidalis. Hin und wieder findet man auch den Bastard Robinia pseudacacia $X$ Robinia glutinosa mit drüsigborstigen Trauben.

Der Same ist nicht bei allen Formen gleichartig, meist ist er von schmutzig brauner Farbe mit feinen schwarzen Sprengeln und seitlich zusammengedrückt, aber man findet auch solche von schwarzbrauner Farbe, die dann den giftigen Goldregensamen in Farbe und Gestalt ähnlich seben, sodaß es bei dem ziemlich gleichen Aussehen der Bäume (Sträucher) beim Einsammeln zu Verwechslungen kommen kann. Aus diesem Grunde sehe ich mich veranlaßt, auch den Samenbau von Cytisus Laburnum zu beschreiben.

Die Samenkörner von Robinia pseudacacia bleiben mit der aufgesprungenen Hülse oft bis ins folgende Jahr an den Zweigen (was das Einsammeln sehr erleichtert), während die Hülsen von Goldregen früher oder später nach der Reife abfallen.

1) Diese Zeitschrift 1918, 35, 275. 


\section{Robinia pseudacacia.}

Sehen wir uns nun den anatomischen Bau der Samen an. Er ist der für die Leguminosenfrüchte typische, der schon oftmals ${ }^{1}$ ) beschrieben wurde. Eine für die praktische Kontrolle hinreichende Bescbreibung des anatomischen Baues der Samenschale aller für das Vorkommen in Nahrungsmitteln in Betracht kommenden Leguminosen findet man in J. Moeller, "Mikroskopie der Nabrungs- und Genußmittel aus dem Pflanzenreich“2). Dieses Buch enthält auch einen analytischen Schlüssel zur Bestimmung der einzelnen Gattungen.

Ich will nun zuerst den anatomischen Bau besonders an Robinia pseudacacia beschreiben, desgleichen den von Cytisus Laburnum, der dem ersteren gegenüber nur ganz geringfügige Unterschiede zeigt.

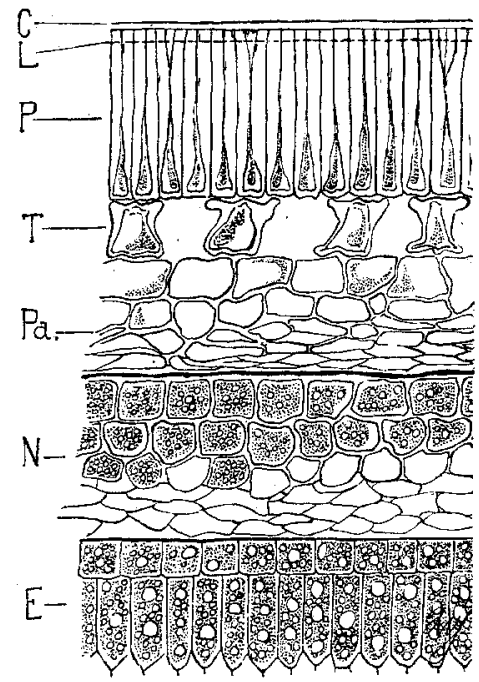

Fig. 1.

Robinia pseudacacia L.

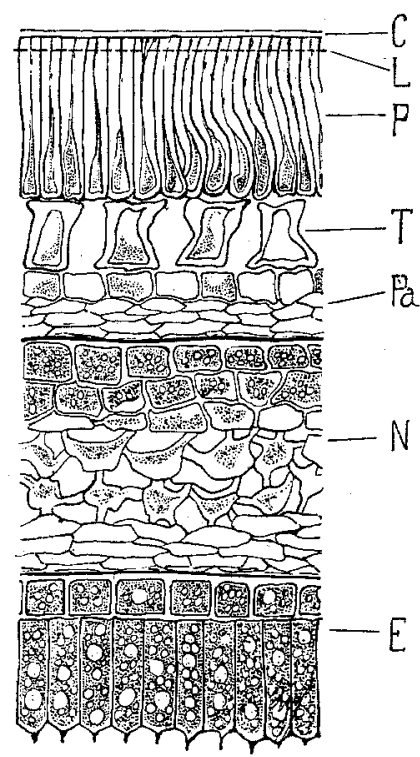

Fig. 2.

Cytisus laburnum

Schnitt durch die Samenschale: ${ }^{*} \mathrm{C}$ Cuticula; L Lichtlinie; P Palisadenschicht; T-Trägerzellenschicht; Pa Parenchym der Samenschale; N Nucellarrest; E Embryo.

Ein Querschnitt durch den Samen (Fig. 1) läßt uns die Samenschale, darunter einen mehr oder weniger zurückgebliebenen Nucellarrest (Perisperm) und den Embryo erkennen.

Die Samenschale setzt sich aus der Palis adenschicht, der Trägerzellen. schjcht und dem Parenchym der Samenschale zusammen.

1) J. Chalon, La graine des Legumineuses; 1. cellules de la carapace, 2. albumen. Mons 1875.

Haberlandt, Über die Entwickelungsgeschichte und den Bau der Samenschale bei der Gattung Phaseolus. - Sitzungslier. d. Wiener Akademie 1877, 75, Januarheft, S. 6.

A. Sempolowski, Inauguraldissertation Leipzig 1874. 1878, 79.

G. Beck, Die Samenschale einiger Leguminosen. - Sitzungsber. d. Wiéner Akademie

2) Verlag von Julius Springer in Berlin. 
Die Palisadenschich t (P) besteht aus zahlreichen säulenförmigen Sklereiden, die eine Länge von $90-115 \mu$ und eine Breite von $8 \mu$ besitzen; sie sind stets ganz gerade, niemals geknickt oder gebogen. Das Zellinnere derselben verengt sich nach außen in einen feinen Kanal; sehr oft gabelt sich dieser noch in 2 oder 3 Kanälchen, namentlich dort, wo die Palisadenzellen infolge einer starken Krümmung der Samenoberfläche nach außen oft bis zum Doppelten an Breite zunebmen. Nach auBen zu sind die Palisaden mit einer dünnen Cuticula (C) überzogen. In einer Entfernung von $5 \mu$ von der Cuticula verläuft die ebenso breite Lichtlinie $\left.{ }^{1}\right)(\mathrm{L})$.

Als zweite Schicht folgen die Trägerzellen (T) in ihrer charakteristischen Gestalt, die Chalon ,cellules en sablier" (Zellen wie eine Sandubr) nannte; sie sind bei Robinia beiläufig $25 \mu$ hoch und in der Mitte $15 \mu$ breit. 'Die Zellenwanddicke derselben nimmt gegen die Palisadenschicht zu und erreicht hier $4 \mu$. Die Interzellularräume $z$ wischen den einzelnen Zellen sind ziemlich groß, jedoch sehr unregelmäßig. Von Absonderung von Gallerte in diesen großen Interzellularen, wie es Schleide $\mathbf{n}^{2}$ ) für die Papilionaceen behauptet, fand sich nichts. Nach innen schließt sich als weitere Schicht das Parenchym der Samenschale $(\mathrm{Pa})$ an in einer beiläufigen Dicke von $130 \mu$. Nur die erste oder zweite Zellreihe davon sind parenchymatisch ausgebildet, gegen das Innere zu pressen sich die einzelnen Zellen einander ganz flach.

Ein anderes Aussehen zeigt die Samenschale in der Gegend der Nabelspalte. $\mathrm{Zu}$ beiden Seiten derselben lagert sich auf die Palisadenschicht, die sich hier beiderseitig hügelartig vorwölbt, eine zweite Palisadenschicht ${ }^{3}$ ), die das entstandene Tal zum größten Teile ausfüllt. Vollständig anders gestaltet sind hier die Trägerzellen, die thre Sanduhrgestalt verlieren und die Form mehr rundlicher Sklereiden annelmen und nicht eine Zellreihe bilden, sondern vielreihig angehäuft sind, wobei sie teilweise das Parenchym der Samenschale durebsetzen. Solche rundlichen Trägerzellen findet man stets, zu kleinen lockeren Nestern vereinigt, in dem gemahlenen Samenpulver.

Durch das Samenhäutchen von der Samenschale getrennt seben wir unter derselben einen ziemlich großen Nucellarrest (Perisperm) $(\mathbf{N})$, der an den Stellen mit starker Oberflächenkrümmung von den sich streckenden und verbreiternden Kotyledonen ganz an die Samenschale gepreßt ist, soda $B$ er bier nur aus flach zusammengedrückten Zellen besteht, die keinerlei Reservestoffe mehr enthalten; dagegen erreicht er an den flachen Teilen des Samens eine. Mächtigkeit von $200 \mu$. Hier enthalten die äußersten Zellreihen auch noch Reservestoffe; nach innen zu nehmen sie ebenfalls eine mehr flach zusammengepreßte Gestalt an.

Die Kotyledonen (E) sind ganz stärkefrei. Thre erste Zellschicht, die Aleuronschicht besteht aus Zellen mit quadratischem Querschnitt, deren Seitenkante beiläufig $12-13 \mu$ mißt. Das sonstige Gewebe setzt sich aus prismatischen Zellen zusammen, die unmittelbar unter der Aleuronschicht 45-55 $\mu$ lang und 18-22 $\mu$ breit sind, gegen das Innere nimmt aber die Länge derselben immer mehr zu und erreicht $80 \mu$, während die Breite die gleiche bleibt.

1) Unter der Lichtlinie versteht man eine Querzone, die durch alle Palisadenzellen länft, in der das Licht stärker gebrochen wird und die dadurch heller erscheint. Vergl. hierzu Innovicz, Die Lichtlinie in den Prismenzellen der Samenschalen, - Sitzungsber. d. Wiener Akademie 1878, 76, 335.

2) Schleiden und J. R. Vogel, Nova acta. d. Leopold.-Carol. Acad. 19, II, 51.

$\left.{ }^{3}\right)$ Diese $z$ weite aufgelagerte Palisadenschicht zeigt keine Lichtlinie, worauf bei der Untersuchung im Pulver ebenfalls zu achten ist. 


\section{Cytisus Laburuum.}

Der anatomische Bau des Samens von Cytisus Laburnum ist dem von Robinia pseudacacia sehr ähnlich und die Unterscheidung durch das Mikroskop im gerösteten Samenpulver sebr schwer. Die kurze Beschreibung und die Abbildung (Fig. 2) sollen das veranschaulichen. Die Palisadenschicht $(\mathrm{P})$ ist $90-100 \mu$ hoch; die einzelnen Zellen sind 8-10 $\mu$ breit und von denen von Robinia pseudacacia insofern unterscheidbar, als viele von ibnen nicht gerade, sondem leicht gebogen sind. Die Trägerzellen (T) sind an Größe und Gestalt nicht verschieden, nur daß die Dicke der Zellenwände nicht so sehr nach auBen zunimmt. Der Nucellarrest $(\mathrm{N})$ ist an den Längsseiten des Samens etwas mächtiger $(270 \mu)$ als bei Robinia; auch bat das Gewebe in seiner Mitte ein lockeres schwammiges Gefüge. Die Zellen der Kotyledonen (E) sind etwas größer, enthalten aber ebenfalls keine Stärke, sondern nur Fett als Reservestóff.

Legt man einen Schnitt durch den Samen in kono. Schwefelsäure, so färbt sich bei Robinia die Samenschale braun, der Keimling rosenrot. Tut man dasselbe bei Cytisus Laburnum, so färbt sich die Samenschale chromgelb und auch die Kotyledonen nehmen eine mebr gelbe Färbung an; sehr oft entstehen auch in diesen sofort gelbe Flecke, die erst allmählich eine rötliche Farbe annehmen. Ich versuchte nun das bei dem Pulver gerösteter Samen anzuwenden und fand die Reaktion auch hier brauchbar, sofern die Samen nicbt alluu stark gebrannt waren; Teilchen, die das nicht sind, findet man übrigens auch in stark gebrannten Pulvern nach längerem Suchen. Besonders gut kann man diese Beobachtung bei Fragmenten der Palisadenschicht in der Flächenaufsicht machen; die einzelnen Palisadenzellen lösen sich von einander los und gruppieren sich sternförmig um das Teilchen. Bei Robinia gelt das Loslösen sehr rasch vor sich und das Fragment erscheint von einem dunkelbraunen Hof umgeben; bei Cytisus geschieht das Loslösen viel langsamer und das Teilchen umgibt sich mit einem deutlich chromgelben Hof. Durch diese Reaktion läbt sich Cytisus im Pulver gerösteter Robiniensamen nachweisen.

Der Akazienbaum gilt in Volke vielfach für giftig, auch in der Literatur findet man vieles, was auf die Giftigkeit desselben hinweist. Kobert zählt in seinem Werke "Die Intoxikationen" eine Reibe an Tieren und Menschen beobachteter Erkrankungen auf, die nach dem Genusse von verschiedenen Teilen des Akazienbaumes eintraten. Pow e r ${ }^{1}$ ), der sich eingehend mit den giftigen Wirkungen der Robinienrinde beschäftigte, fand in dieser neben Gerbsäure, Zucker, Fett, dem Glycosid Siringin und Siringinsäure ein Nucleoproteid, dem er den Namen „Robin“ gab. Dieses ist in Wasser und Salzlösungen löslich und durch Säuren ausfällbar. Es koaguliert beim Erhitzèn und wird dadurch entgiftet. Das "Robin" gibt alle Reaktionen der Eiweibkörper und wird durch alle Eiweißreagentien gefällt. Pow er läßt jedoch die Frage offen, ob es durch diese wirklich gefällt oder nur von den Eiweilkörpern mitgerissen wird.

Kobert und Weinhaus ${ }^{2}$ ) fanden in den Samen und Rinden verschiedener Pflanzen namentlich aus der Familie der Papilionaceen und aus der Solanaceengattung Datura eigenartige Stoffe, denen sie den Namen Phasine gaben. Es sind das stickstoffhaltige Substanien, die die Eigenschaft haben, beim Vermischen mit roten Blutkörperchen auf diese agglutinierend (verklebend) zu wirken, aber - mit

1) Pharm. Journ, 1901, 67, 258 u. 275.

2) Biochem. Zeitschr. 1909, 18, Heft 3-5; vergl. auch Kobert, Chemiker-Ztg. 1918, 42, 246 . 
Ausnahme des Rhizins und des Abrins - innerlich ungiftig sind. Auch das "Robin" gehört zu dieser Gruppe und zwar zu den Rindenphasinen, Kobert erklärt es, obwohl er es früher für giftig hielt, in neuerer Zeit für innerlich ungiftig, während Power an der Giftigkeit desselben noch jetzt festhält.

Ich stellte Auszüge von Robinienrinde und Robiniensamen mit physiologischer Kochsalzlösung durch zweistündiges Einwirkenlassen derselben bei $40^{\circ}$ dar. Beide Auszüge wirkten sowohl auf das Blut von Kaninchen als auch auf das des Menschen agglutinierend. Beim Kochen fiel in dem Rindenauszug ein feinflockiger weißer Niederschlag aus, in dem Samenauszug, der mebr Eiweißstoffe gelöst enthielt, ein gelblicher Niederschlag in grolen Flocken. Der Rindenauszug wirkte nath dem Kochen nur äuBerst schwach, der Samenauszug überhaupt nicht agglutinierend. Der Samen von Robinia pseudacacia enthält also ebenfalls ein thermolabiles Phasin, das möglicherweise mit dem Rindenphasin "Robin" identisch ist. Durch Aceton läßt es sich aus der wässerigen Lösung ausfällen.

Die Fütterungsversuche von L. H. van Berk ergaben, daf Robiniensamen für Mäuse innerlich ungiftig sind, was auch von $\mathrm{C}$. Griebel bestätigt werden konnte. Ich selbst habe eine Abkochung von $20 \mathrm{~g}$ gerösteter Samen mit 200 cem Wasser, ohne die geringsten Wirkungen auf meinen Organismus zu spüren, getrunken. Der Geschmack derselben ist ziemlich kaffeeähnlich, die Färbekraft des gerösteten Samens jedoch gering. Dagegen möchte ich, zurückgreifend auf die im Anfang erwähnte Notiz in der Zeitschrift für angewandte Chemie, es bezweifeln, daf die Robiniensamen einen Kakaoersatz liefern. Man kann bekanntlich nicht zwei Herren gleichzeitig dienen. Im ersten Augenblick schmeckt die Abkochung zwar kakaoähnlich bitter, man braucht aber nur etwas Zucker gleichzeitjg auf die Zunge zu bringen, um zu merken, daß es den Kakao bei weitem nicht ersetzen kann. Durch die Entfernung des Fettes jer Samen enthält 13,30 fettes Ol, bestehend aus Glyceriden der Stearin-, Eruca-, Öl-, Linol- und Linolensäure - vor dem Rösten gewinnt das Ersatzmittel sowohl an Geschmack als auch an Färbekraft.

Die Frage, ob der Genuß von aus Robiniensamen hergestellten Nahrungs- und Genubmitteln dem Menschen auch auf die Dauer unschädlich ist, wird man erst dann zu beantworten imstande sein, wenn die neuen Ersatzstoffe längere Zeit im Verkehr waren und man sich auf etwaige besondere Fälle aus der Praxis wird stützen können. Der Zweck meiner Untersuchung war es, den Untersuchungsanstalten die gewiß schwierige Stellungnahme zu neu auftauchenden Ersatzstoffen wenigstens einigermaßen zu erleichtern. 